\title{
MicroRNA-204 targets signal transducer and activator of transcription 5 expression and inhibits proliferation of B-cell lymphoma cells
}

\author{
$\mathrm{LI} \mathrm{WU}^{1^{*}}$, YONG LI $^{1 *}$, JIAN-MIN FAN $^{2 *}$, ZHI-MIN ZHANG ${ }^{2}$, \\ JIN-LING OUYANG ${ }^{1}$, TING-TING NI ${ }^{1}$, HAI-XIA WU ${ }^{1}$ and HANG LI ${ }^{1}$ \\ ${ }^{1}$ Department of Oncology, People's Hospital of Guizhou Province, Guiyang, Guizhou 550002; \\ ${ }^{2}$ Department of Hematology, Guiyang College of Medicine, Guiyang, Guizhou 550004, P.R. China
}

Received January 2, 2014; Accepted October 23, 2014

DOI: $10.3892 / \mathrm{mmr} .2015 .3298$

\begin{abstract}
The function of microRNAs (miRNAs) in tumorigenesis has been extensively investigated. In the present study, the aim was to investigate the expression and role of miR-204 in B-cell lymphoma. The present study demonstrated that miR-204 is downregulated in B-cell lymphoma. Using in vitro studies, overexpression of miR-204 was shown to inhibit growth in Daudi and Raji B-cell lymphoma cell lines. Furthermore, miR-204 could bind the 3'-untranslated region of signal transducer activator of transcription 5 (STAT5), a transcription factor that promotes B-cell lymphoma oncogenesis. Re-introduction of STAT5 reversed the antiproliferative roles of miR-204, confirming the specific importance of STAT5 for miR-204 action in cell proliferation. The present study suggests a novel mechanism for dysregulated miRNAs in the progression of B-cell lymphoma.
\end{abstract}

\section{Introduction}

There are numerous types of B-cell lymphoma, including: Burkitt lymphoma, chronic lymphocytic leukemia, diffuse large B-cell lymphoma, mantle cell lymphoma, follicular lymphoma, marginal zone lymphoma/mucosa-associated lymphoid tissue lymphoma, nodal marginal zone B-cell lymphoma, splenic marginal zone lymphoma and other less frequent malignancies $(1,2)$. The pathogenic mechanisms of B-cell lymphoma remain largely unknown; therefore, further

Correspondence to: Dr Hang Li, Department of Oncology, People's Hospital of Guizhou Province, 83 Zhongshan Road, Guiyang, Guizhou 550002, P.R. China

E-mail: hangligz@foxmail.com

\section{*Contributed equally}

Key words: microRNA-204, signal transducer and activator of transcription 5, B-cell lymphoma, proliferation investigation may aid in improving the accuracy of lymphoma diagnosis and gene therapy.

Previous studies have demonstrated the importance of microRNAs (miRNA), a type of non-coding small RNA, in cell proliferation, apoptosis and differentiation $(3,4)$. Altered miRNA expression has also been suggested in lymphoma development and progression (5-7). A transgenic mouse model with conditional overexpression of miR-155 in B-lymphocytes, exhibited polyclonal pre-leukemic pre-B-cell proliferation, followed by mature B-cell malignancy (8). In addition, miR-155 has been shown to have prognostic implications in a subtype of human Non-Hodgkin lymphoma and diffuse large B-cell lymphoma (9). These findings suggest that aberrant expression of certain miRNAs may be a potential biomarker for the diagnosis of lymphoma.

In the present study a miRNA expression screen was conducted using B-cell lymphoma and non-tumoral samples, in order to observe the expression of different miRNAs and determine whether any are involved in the development of B-cell lymphoma.

\section{Materials and methods}

Human samples. A total of 22 fresh-frozen samples of B-cell lymphoma and 16 non-tumoral samples (reactive lymph nodes) were collected. A total of 38 samples were collected at the People's Hospital of Guizhou Province (Guiyang, China) between 2009 and 2012. The non-tumoral samples were collected from different patients. All lymphoma samples were obtained patients who had been diagnosed and were obtained prior to the patients receiving therapy. The project was approved by the Ethics Committee of the People's Hospital of Guizhou Province.

Cell culture. The Daudi and Raji B-cell lymphoma cell lines were obtained from American Type Culture Collection (Manassas, VA, USA). The cells were cultured in Dulbecco's modified Eagle's medium (Gibco Life Technologies, Beijing, China) supplemented with $10 \%$ fetal bovine serum (Gibco Life Technologies). The cultures were maintained at $37^{\circ} \mathrm{C}$ in a humidified atmosphere containing $5 \% \mathrm{CO}_{2}$. 
Analysis of miRNA expression using TaqMan ${ }^{\circledR}$ reverse transcription-quantitative polymerase chain reaction $(R T-q P C R)$. Total RNA from the tissue samples and cell lines was extracted using the miRNA Isolation kit (Ambion Life Technologies, Carlsbad, CA, USA). The expression levels of mature miRNAs were assayed using a TaqMan ${ }^{\circledR}$ MicroRNA assay, specific for hsa-miR-204 (Applied Biosystems Life Technologies, Foster City, NJ, USA). Briefly, 10 ng total RNA was reverse transcribed into cDNA using specific stem-loop RT primers. The primer sequences were as follows: p21, forward 5'-TGTCCGTCAGAACCCATGC-3' and reverse 5'-AAAGTCGAAGTTCCATCGCTC-3'; p27, forward 5'-AGGAGGAGATAGAAGCGCAGA-3' and reverse 5'-GTGCGGACTTGGTACAGGT-3'; Cyclin A, forward 5'-CGCTGGCGGTACTGAAGTC-3' and reverse 5'-GAGGAACGGTGACATGCTCAT-3'; Cyclin B1, forward 5'-AATAAGGCGAAGATCAACATGG-3' and reverse 5'-TTTGTTACCAATGTCCCCAAGAG-3'; Cyclin D1, forward: 5'-GCTGCGAAGTGGAAACCATC-3' and reverse 5'-CCTCCTTCTGCACACATTTGAA-3'. All primers were provided by Biosune (Shanghai, China). The qPCR was performed using an Applied Biosystems 7900 Real-time PCR system (Life Technologies, Grand Island, NY, USA) and a TaqMan ${ }^{\circledR}$ Universal PCR Master mix (TaKaRa Bio Inc., Dalian, China). All of the primers were obtained from the TaqMan miRNA assays. Small nuclear U6 snRNA (Applied Biosystems Life Technologies) was used as an internal control. PCR conditions included an initial holding period at $94^{\circ} \mathrm{C}$ for 5 min, followed by a two-step PCR program consisting of $94^{\circ} \mathrm{C}$ for $5 \mathrm{sec}$ and $60^{\circ} \mathrm{C}$ for $30 \mathrm{sec}$ for 45 cycles. Relative quantification analysis of gene expression analysis was performed according to the $2^{-\Delta \Delta}$ method.

Transfection. miR-204 mimics, antisense oligonucleotides and negative controls were obtained from GenePharma, Co., Ltd. (Shanghai, China). For the transient transfections, a complex of Lipofectamine ${ }^{\circledR} 2000$ (Invitrogen Life Technologies) and $25 \mathrm{~nm}$ miRs was prepared, according to the manufacturer's instructions. For the STAT5 re-introduction experiments, Daudi or Raji cells were pre-transfected with miR-204 mimics or negative control (NC) for $24 \mathrm{~h}$, and then transfected with STAT5 expression plasmids or empty vector (EV) for another $30 \mathrm{~h}$.

Bromodeoxyuridine (BrdU) assays. A cell proliferation ELISA (BrdU kit; Beyotime Institute of Biotechnology, Haimen, China) was used to analyze the incorporation of BrdU during DNA synthesis, according to the manufacturer's instructions. All of the experiments were performed in triplicate. Absorbance was measured at $450 \mathrm{~nm}$ using a Spectra Max 190 ELISA reader (Molecular Devices, Sunnyvale, CA, USA).

Western blotting. The cells or tissues were harvested and lysed with ice-cold lysis buffer $(50 \mathrm{~mm}$ Tris- $\mathrm{HCl}, \mathrm{pH} 6.8$, $100 \mathrm{~mm} 2-\mathrm{ME}, 2 \% \mathrm{w} / \mathrm{v}$ SDS and $10 \%$ glycerol). Following centrifugation at $20,000 \mathrm{x} \mathrm{g}$ for $10 \mathrm{~min}$, the proteins in the supernatants were quantified. The proteins were separated by $10 \%$ SDS PAGE and transferred to nitrocellulose membranes (GE Healthcare Life Sciences, Chalfont, UK). The membranes were then blocked with $10 \%$ nonfat milk in phosphate-buffered saline, followed by an incubation with the primary antibodies indicated. The membranes were then further incubated with horseradish peroxidase-linked secondary antibodies (Cell Signaling Technology, Inc., Danvers, MA, USA). The signals were detected using SuperSignal West Pico Chemiluminescent Substrate kit (Pierce Biotechnology, Inc., Rockford, IL, USA), according to the manufacturer's instructions. Anti-signal transducer and activator of transcription 5 (STAT5; rabbit polyclonal antibody targeting human STAT5, cat no. ab7969, $1: 2,000$ ) and $\beta$-actin (mouse monoclonal antibody targeting human $\beta$-actin, cat no. ab6276, 1:1,000) were purchased from Abcam (Cambridge, MA, USA). The protein expression levels were normalized to total $\beta$-actin. The potential target genes of miR-204 were predicted by miRWalk software (http://www. umm.uni-heidelberg.de/apps/zmf/mirwalk/).

Luciferase reporter assay. Total cDNA from the Daudi cells was used to amplify the 3 '-untranslated region (3'-UTR) of STAT5 by PCR. The STAT5 3'-UTR was cloned into the pMIR-Report vector carrying firefly luciferase (Ambion Life Technologies), yielding pMIR-Report-STAT5. Mutations were introduced into the potential miR-204 binding sites, using the QuikChange Site-Directed Mutagenesis kit (Agilent Technologies, Inc., Santa Clara, CA, USA). The cells were transfected with the pMIR-Report vectors containing the 3'-UTR variants and miR-100 precursor control plasmids for $36 \mathrm{~h}$. The pRL-TK vector (Promega Corporation, Madison, WI, USA) carrying the Renilla luciferase gene was used as an internal control to normalize the transfection efficiency. Luciferase values were determined using the Dual-Luciferase Reporter Assay system (Promega Corporation).

miRNA microarray. miRNA microarrays were performed by Kangchen (Shanghai, China) using Human miRNA microarray chips (Release 16.0, 8x60K; Agilent Technologies).

Cell cycle analysis. For cell cycle analysis, propidium iodide (PI) was used to identify the proportion of cells in each of the three interphase stages of the cell cycle. Cells were harvested and fixed in $1 \mathrm{ml}$ cold $70 \%$ ethanol overnight at $-20^{\circ} \mathrm{C}$. DNA was stained in PI/RNaseA solution and the DNA content was detected using flow cytometry. Data was analyzed using WinMDI 2.8 software (freeware from Joe Trotter, La Jolla, CA, USA).

Statistical analysis. Data are expressed as the mean \pm standard error of the mean from $\geq 4$ separate experiments. Differences between the groups were analyzed using Student's t-test or one-way analysis of variance. Statistical analyses were performed with Prism GraphPad software (Version 5.01) (GraphPad Software Inc., La Jolla, CA, USA). P $<0.05$ was considered to indicate a statistically significant difference.

\section{Results}

miR-204 expression levels are decreased in B-cell lymphoma. To identify novel miRNAs that are dysregulated in B-cell lymphoma, a 'miRNome' expression profile was performed, using miRNA microarrays, on RNA isolated from freshly frozen B-cell lymphoma and non-tumoral samples. The screen 
A

List of mRNAs downregulated in Lymphoma $(p<0.01)$.

\begin{tabular}{lcc}
\hline miRNA & p-Value & $x$-Fold $(\mathrm{C} / \mathrm{N})$ \\
miR-204 & 0.00119 & 0.2218 \\
miR-192 & 0.00283 & 0.4927 \\
miR-411 & 0.00351 & 0.3743 \\
miR-801 & 0.00579 & 0.4298 \\
miR-27b & 0.00448 & 0.6782 \\
miR-15a & 0.00188 & 0.5504 \\
miR-132 & 0.00920 & 0.4217 \\
miR-149 & 0.02652 & 0.3958
\end{tabular}

B

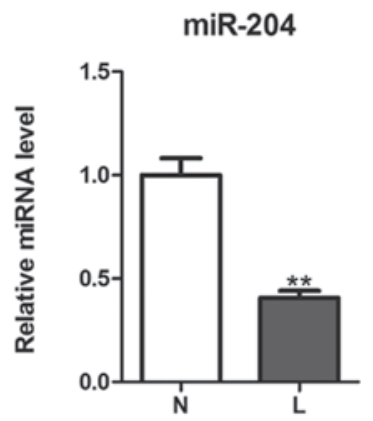

Figure 1. Downregulation of microRNA (miR)-204 in B-cell lymphoma samples. (A) List of miRNAs downregulated in B-cell lymphoma (L) and non-tumoral tissues $(\mathrm{N})(\mathrm{P}<0.01)$. Microarray analysis was performed in quadruplicate and the mean \pm standard error is indicated. $(\mathrm{B})$ miR-204 expression levels were determined by TaqMan ${ }^{\circledR}$ quantitative polymerase chain reaction in $\mathrm{L}$ and $\mathrm{N}$ tissues. ${ }^{* *} \mathrm{P}<0.01$, compared with $\mathrm{N}$.

A

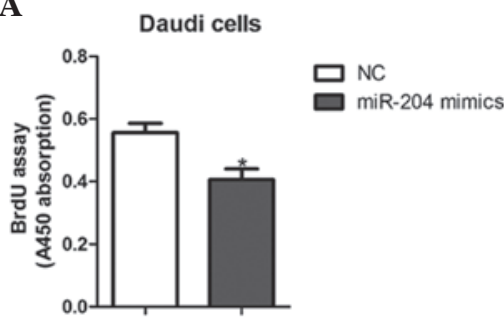

D

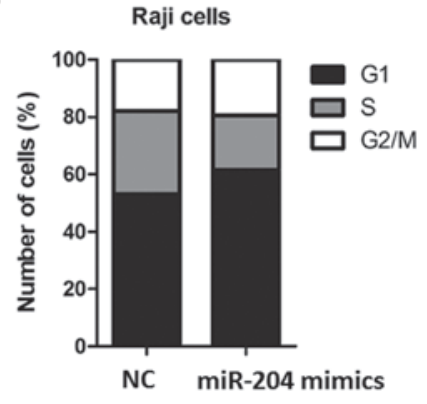

B

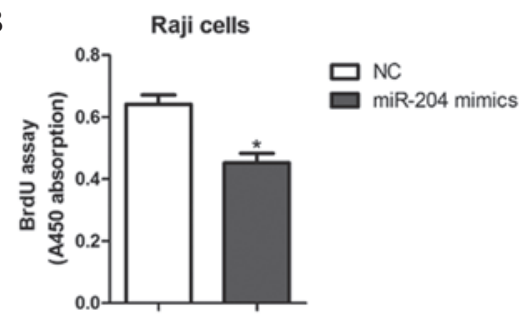

$\mathbf{E}$

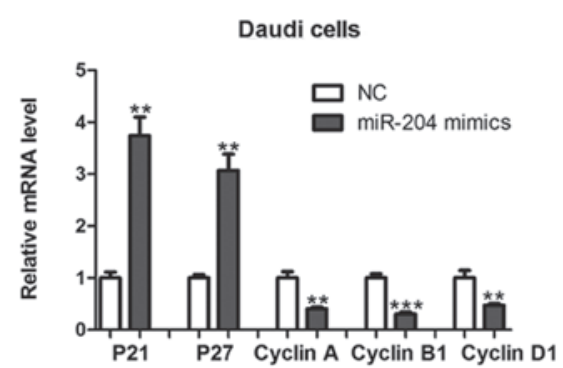

C

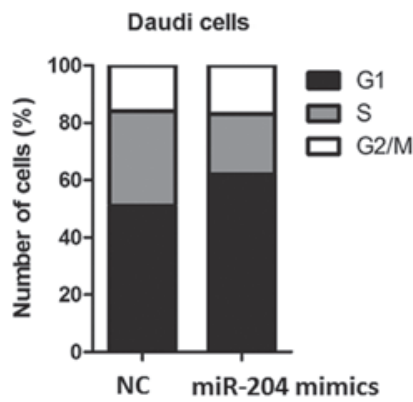

$\mathbf{F}$

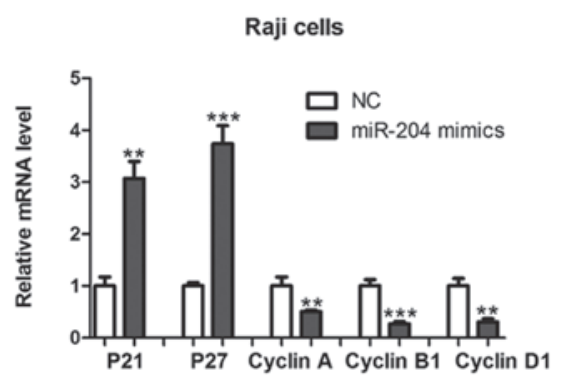

Figure 2. Transfection of cells with miR-204 mimics inhibits cell proliferation. (A and B) The cell proliferative potential was determined in (A) Daudi and (B) Raji B-cell lymphoma cells transfected with miR-204 mimics or negative control (NC), as determined by a bromodeoxyuridine (BrdU) assay. A450 absorption was assayed $30 \mathrm{~h}$ post-transfection. (C and D) Cell cycle distribution of the (C) Daudi and (D) Raji cells transfected with miR-204 mimics or NC was analyzed by flow cytometry. The cells were labeled for $20 \mathrm{~min}$ with propidium iodide and immediately analyzed by flow cytometry. Histograms represent the percentage of cells in each phase of the cell cycle $\left(\mathrm{G}_{0} / \mathrm{G}_{1}, \mathrm{~S}\right.$ and $\left.\mathrm{G}_{2} / \mathrm{M}\right)$. (E and F) Quantitative polymerase chain reaction was used to determined expression levels of p21, p27, and cyclins A, B1 and D1 in (E) Daudi and (F) Raji cells transfected with miR-204 mimics or NC for $36 \mathrm{~h}$. $\mathrm{P}<0.05 ;{ }^{* * *} \mathrm{P}<0.01$; **** $\mathrm{P}<0.001$, compared with NC. Error bars indicate standard error of the mean.

revealed a pronounced downregulation in the expression levels of miR-204 in B-cell lymphoma tissues ( $\mathrm{P}<0.01$; Fig. 1A). Decreased miR-204 expression levels were further confirmed by qPCR (Fig. 1B). These results are the first, to the best of our knowledge, to demonstrate that the expression levels of miR-204 are significantly reduced in B-cell lymphoma tissue.

Transfection with miR-204 mimics inhibits cell proliferation. To assess the effects of miR-204 on B-cell lymphoma growth, miR-204 mimics or NCs were transfected into Daudi and Raji cells. Transfection with the miR-204 mimics inhibited the proliferative ability of the cells, as evidenced by BrdU incorporation assays (Fig. 2A and B). Furthermore, miR-204 mimics significantly increased the percentage of cells in the $\mathrm{G}_{0} / \mathrm{G}_{1}$ phase and decreased the percentage of cells in the $\mathrm{S}$ phase (Fig. 2C and D).

The present study also determined whether the inhibition of cell proliferation was associated with the expression of the genes that regulate the $G_{1} / S$ transition. These include the cyclin-dependent kinase (CDK) inhibitors p21 and p27, and the CDK regulators cyclins $\mathrm{A}, \mathrm{B} 1$ and D1. The expression levels of p21 and p27 were increased, whereas the expression 
A

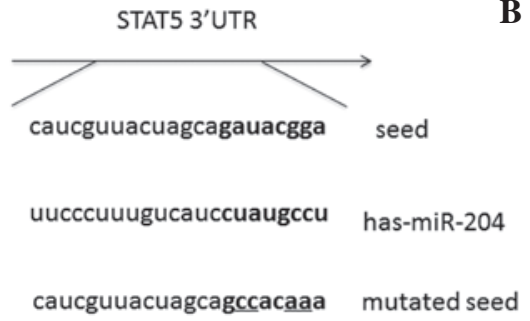

D

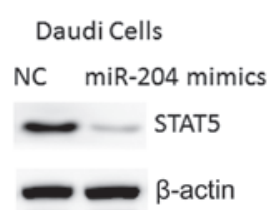

B

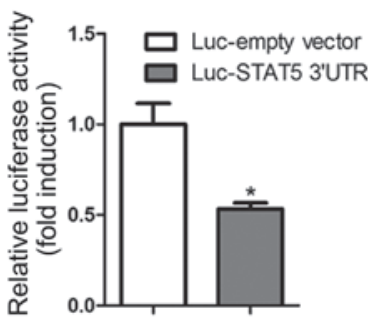

$\mathbf{E}$

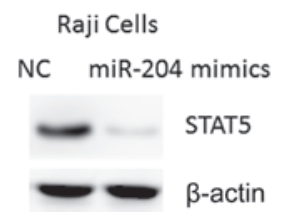

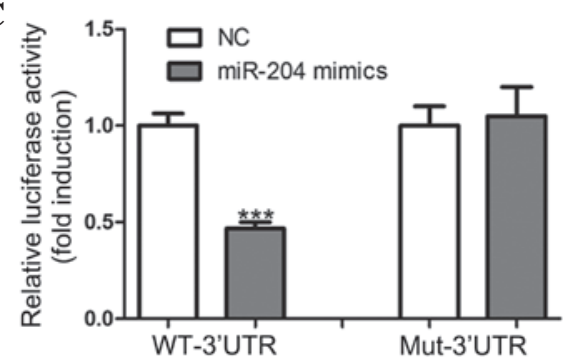

F $\mathrm{N}$ L

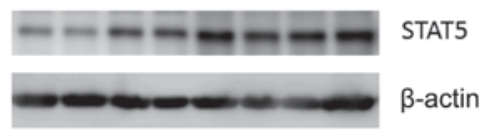

Figure 3. MicroRNA (miR)-204 negatively regulates signal transducer and activator of transcription 5 (STAT5) protein by targeting its 3'-untranslated region (UTR). (A) Graphic representation of miR-204 binding sites in the 3'-UTR of the human STAT5 gene. The mutated seed is underlined. (B) Relative luciferase activity of the firefly luciferase reporter constructs containing either an empty vector or 3'-UTR of the human STAT5 gene. (C) Luciferase reporter assays in Daudi B-cell lymphoma cells. The cells were transfected with $100 \mathrm{ng}$ wild-type 3'-UTR-reporter (WT) or mutant (Mut) constructs, together with $24 \mathrm{~nm}$ miR-204 mimics or negative controls (NC). (D and E) Western blot analysis of STAT5 protein expression levels in (D) Daudi and (E) Raji cells transfected with miR-204 mimics or NC for 48 h. (F) Representative western blot analysis of STAT5 protein expression levels in human B-cell lymphoma (L) and non-tumoral samples $(\mathrm{N}) .{ }^{*} \mathrm{P}<0.05$ and ${ }^{* * *} \mathrm{P}<0.001$, compared with NC. Error bars indicate standard error of the mean.

A

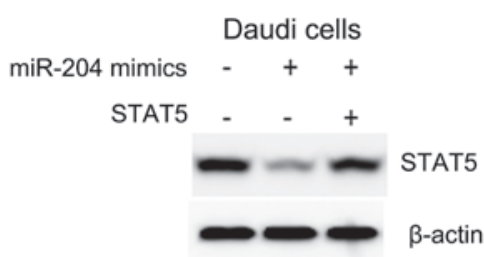

C

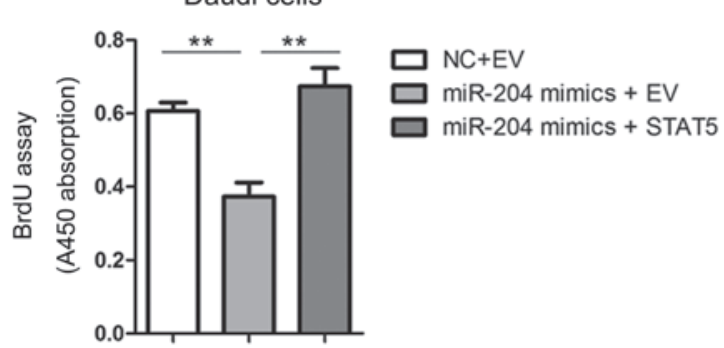

B

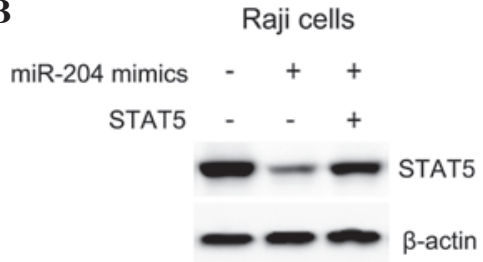

D

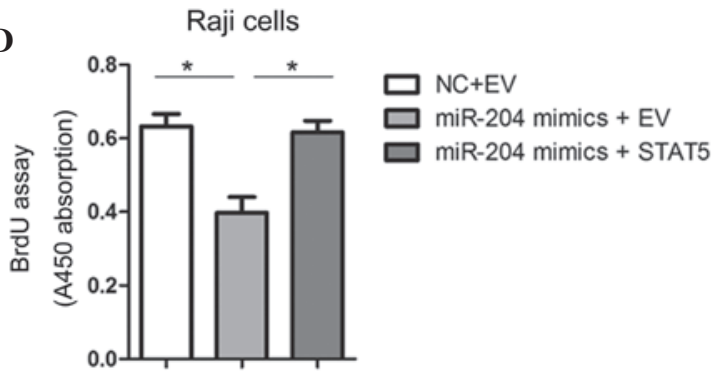

Figure 4. Re-introduction of signal transducer and activator of transcription 5 (STAT5) reverses the antiproliferative effects of microRNA (miR)-204. (A and B) STAT5 protein expression levels were determined by western blotting in (A) Daudi and (B) Raji B-cell lymphoma cells. The cells were pre-transfected with miR-204 mimics or negative control (NC) for $24 \mathrm{~h}$, then transfected with expression plasmids containing STAT5 or empty vector (EV) for a further 30 h. (C and D) The cell proliferative potential was determined using a bromodeoxyuridine (BrdU) assay in (C) Daudi and (D) Raji cells. A450 absorption was assayed $30 \mathrm{~h}$ post-transfection with STAT5 or EV. Error bars indicate standard error of the mean.

levels of cyclins A, B1 and D1 were decreased in the Daudi and Raji cells transfected with the miR-204 mimics, as compared with the NC-transfected cells (Fig. 2E and F).

miR-204 directly regulates STAT5 expression in B-cell lymphoma cells. An aim of the present study was to explore the molecular mechanisms of miR-204 regulation of cell proliferation. A bioinformatics approach (miRWalk) was used to identify numerous putative human miR-204 target genes (data not shown). The gene encoding STAT5 was identified as harboring a potential miR-204 binding site (Fig. 3A) in its 3'-UTR. In Daudi cells, transfection with the luciferase reporter containing the STAT5 3'-UTR resulted in reduced luciferase activity, as compared with the cells transfected with the reporter lacking the STAT5 3'-UTR (Fig. 3B). Furthermore, overexpression of miR-204 led to a reduction in luciferase activity (Fig. 3C). Conversely, mutation of the miR-204 binding motif abrogated the reduced luciferase expression (Fig. 3C). Transfection of the cells with miR-204 mimics led to a reduction in the endogenous protein expression levels of STAT5 in the Daudi and Raji cells (Fig. 3D and E), whereas its mRNA expression levels remained unchanged (data not shown). These results suggest that miR-204 may regulate STAT5 expression at the translational level. Concordantly, the protein expression levels 
A

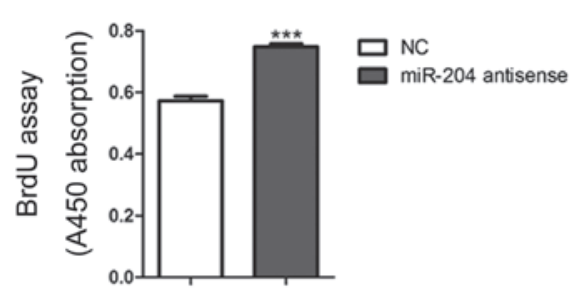

C

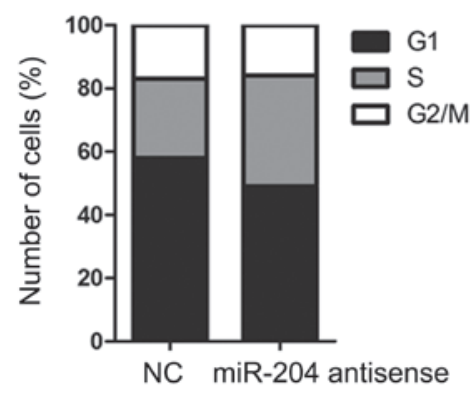

$\mathbf{E}$

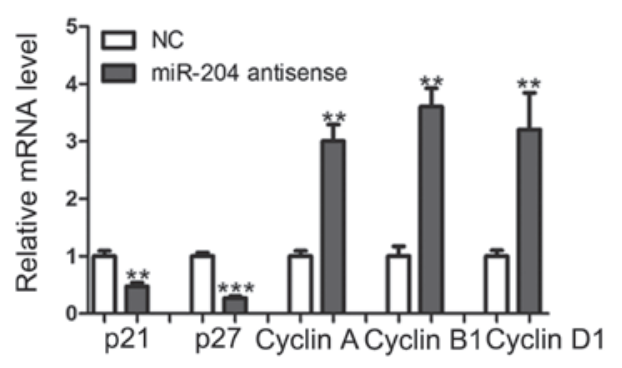

G

Daudi cells

NC miR-204 antisense

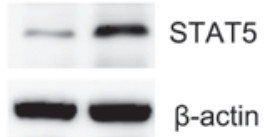

B

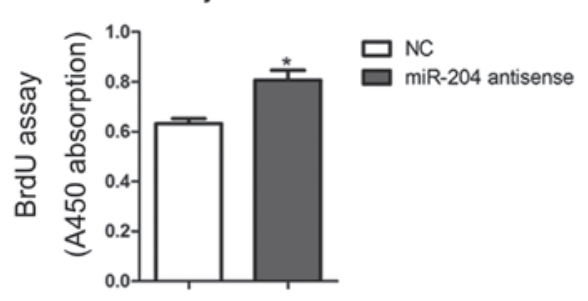

D

Raji cells

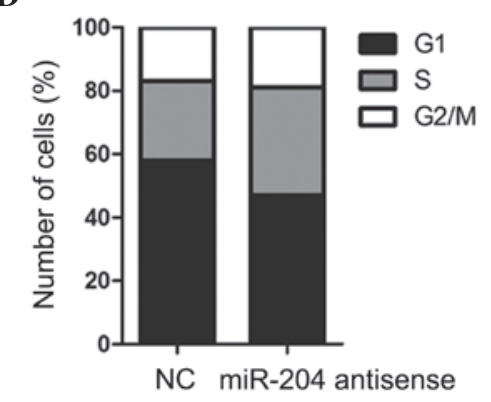

$\mathbf{F}$

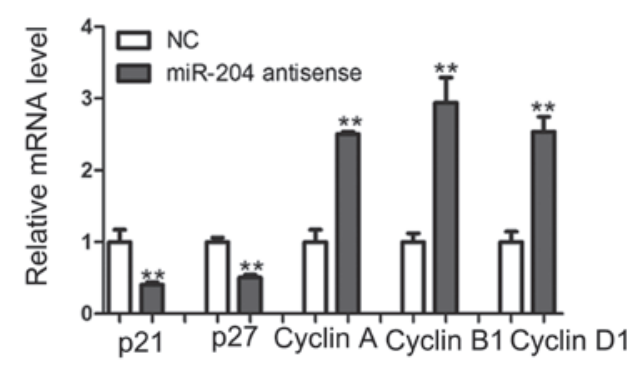

H

Raji cells

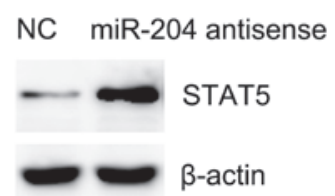

Figure 5. MicroRNA (miR)-204 antisense inhibits the proliferation of B-cell lymphoma cells. (A and B) The cell proliferative potential (BrdU) was determined in Daudi (A) and Raji (B) cells transfected with miR-204 antisense or negative control (NC). A450 absorption was assayed after transfection for $30 \mathrm{~h}$. The cell cycle phase of Daudi (C) and Raji (D) cells transfected with miR-204 antisense or negative control (NC) were analyzed by flow cytometry. Quantitative real-time PCR analysis of p21, p27, Cyclin A, Cyclin B1 and Cyclin D1 in Daudi (E) and Raji (F) cells transfected with miR-204 antisense or negative control (NC) for $36 \mathrm{~h}$. Western blot analysis of STAT5 in Daudi (G) and Raji (H) cells transfected with miR-204 antisense or negative control (NC) for $48 \mathrm{~h}$. "P<0.05; ${ }^{* *} \mathrm{P}<0.01 ;{ }^{* * *} \mathrm{P}<0.001$, compared with NC. Error bars indicate standard error of the mean.

of STAT5 were also upregulated in B-cell lymphoma tissue samples, as compared with the non-tumoral tissues (Fig. 3F).

To verify the functional association between miR-204 and STAT5, the Daudi and Raji cells were transfected with STAT5 expression plasmids, following transfection with miR-204 mimics (Fig. 4A and B). Re-introduction of STAT5 reversed the antiproliferative effects of miR-204, confirming the specific importance of STAT5 for miR-204 action in cell proliferation (Fig. 4C and D). These results suggest that STAT5 is an important target gene of miR-204 in the B-cell lymphoma.

Inhibition of miR-204 promotes the proliferation of $B$-cell lymphoma. As described above, miR-204 has a negative role on the proliferation of B-cell lymphoma. However, it remains unknown whether inhibiting miR-204 promotes cell proliferation. Therefore, the Daudi and Raji cells were transfected with miR-204 antisense oligonucleotides. The ectopic expression of hsa-miR-204 antisense enhanced the proliferative ability of the two cell lines, as compared with the NC-transfected cells (Fig. 5A and B). Furthermore, overexpression of antisense miR-204 resulted in a significantly lower percentage of cells in the $G_{1} / G_{0}$ phase and an increased percentage of cells in the $\mathrm{S}$ phase, as compared with the NC-transfected cells (Fig. 5C and D). In concordance with these findings, the expression levels of p21 and p27 were downregulated, whereas the expression levels of cyclins A, B1 and D1 were upregulated 
in the miR-204 antisense-transfected cells (Fig. 5E and F). Furthermore, the protein expression levels of STAT5 were increased in response to miR-204 inhibition (Fig. 5G and H), further validating STAT5 as a miR-204 target.

\section{Discussion}

The present study demonstrated that miR-204 expression is downregulated in B-cell lymphoma tissue. Forced overexpression of miR-204 was able to inhibit cell proliferation in Daudi and Raji cells, whereas the inhibition of miR-204 by antisense oligonucleotides enhanced cell proliferation. The present study is the first, to the best of our knowledge, to identify miR-204 as a potential tumor suppressor in the progression of B-cell lymphoma. However, in vivo studies are required to further establish this notion.

Initial studies demonstrated that miR-204 has an important physiological role in normal cells, particularly in developmental events and autophagy. miR-204 was previously shown to regulate mesenchymal progenitor cell differentiation, by targeting the 3'-UTR of Runt-related transcription factor 2 (10). Furthermore, miR-204 is essential for the maintenance of the epithelial barrier function, through regulation of transforming growth factor- $\beta$ receptor 2 and SNAIL2 (11). miR-204 may also regulate numerous aspects of eye development in the medaka fish (12). Ablation of miR-204 expression resulted in an eye phenotype characterized by microphthalmia, abnormal lens formation and altered dorsoventral patterning of the retina (12). Subsequent reports have indicated that miR-204 expression is downregulated in numerous types of human cancer (13). Dysregulation of miR-204 has also been shown to mediate migration and invasion of endometrial cancer, by regulating forkhead box $\mathrm{C} 1$ (14). In gastric cancer, downregulation of miR-204 has been reported as having prognostic value and correlates with increased staining of B-cell lymphoma 2 protein in tumoral specimens (15). Furthermore, ectopic expression of miR-204 inhibits colony forming ability, migration and tumor engraftment of gastric cancer cells (15). In glioma, loss of miR-204 expression has been demonstrated to enhance cell migration and stem cell-like phenotype (16).

At the molecular level, the present study identified the transcription factor STAT5 as a potential target of miR-204 function in B-cell lymphoma. This was supported by the observation that miR-204 could bind and inhibit the activity of STAT5 3'-UTR; miR-204 mimics reduced and antisense miR-204 increased the protein expression levels of STAT5, respectively; and re-introduction of STAT5 successfully reversed the antiproliferative roles of miR-204. STAT5 is one of seven members of the STAT family of proteins and has a key role in the generation of B-cell precursors (17). Persistent STAT5 activation is oncogenic, leading to the late emergence of clonal B-cell lymphoma/leukemia $(18,19)$. Therefore, understanding the precise role of the miR-204/STAT5 regulatory axis will advance our knowledge of lymphoma biology, which may be beneficial for its treatment.

\section{Acknowledgements}

The present study was supported by the Guizhou Province Science and Technology Fund Projects [grant no. QKH J (2009) 2214].

\section{References}

1. Seton-Rogers S: Lymphoma: Epigenetic therapy gains momentum. Nat Rev Cancer 12: 798, 2012.

2. Young RM and Staudt LM: Targeting pathological B cell receptor signalling in lymphoid malignancies. Nat Rev Drug Discov 12: 229-243, 2013.

3. Ameres SL and Zamore PD: Diversifying microRNA sequence and function. Nat Rev Mol Cell Biol 14: 475-488, 2013.

4. Sun K and Lai EC: Adult-specific functions of animal microRNAs. Nat Rev Genet 14: 535-548, 2013.

5. Lawrie CH: MicroRNA expression in lymphoma. Expert Opin Biol Ther 7: 1363-1374, 2007

6. Wang LQ, Liang R and Chim CS: Methylation of tumor suppressor microR NAs: lessons from lymphoid malignancies. Expert Rev Mol Diagn 12: 755-765, 2012.

7. Lawrie CH: MicroRNAs and lymphomagenesis: a functional review. Br J Haematol 160: 571-581, 2013

8. Sandhu SK, Volinia S, Costinean S, et al: miR-155 targets histone deacetylase 4 (HDAC4) and impairs transcriptional activity of B-cell lymphoma 6 (BCL6) in the E $\mu$-miR-155 transgenic mouse model. Proc Natl Acad Sci USA 109: 20047-20052, 2012.

9. Ralfkiaer U, Hagedorn PH, Bangsgaard N, et al: Diagnostic microRNA profiling in cutaneous T-cell lymphoma (CTCL). Blood 118: 5891-5900, 2011.

10. Huang J, Zhao L, Xing L and Chen D: MicroRNA-204 regulates Runx2 protein expression and mesenchymal progenitor cell differentiation. Stem Cells 28: 357-364, 2010.

11. Wang FE, Zhang C, Maminishkis A, et al: MicroRNA-204/211 alters epithelial physiology. FASEB J 24: 1552-1571, 2010.

12. Conte I, Carrella S, Avellino R, et al: $\mathrm{miR}-204$ is required for lens and retinal development via Meis2 targeting. Proc Natl Acad Sci USA 107: 15491-15496, 2010.

13. Paulin R, Courboulin A, Barrier M and Bonnet S: From oncoproteins/tumor suppressors to microRNAs, the newest therapeutic targets for pulmonary arterial hypertension. J Mol Med (Berl) 89: 1089-1101, 2011.

14. Chung TK, Lau TS, Cheung TH, et al: Dysregulation of microRNA-204 mediates migration and invasion of endometrial cancer by regulating FOXC1. Int J Cancer 130: 1036-1045, 2012.

15. Sacconi A, Biagioni F, Canu V, et al: miR-204 targets Bcl-2 expression and enhances responsiveness of gastric cancer. Cell Death Dis 3: e423, 2012.

16. Ying Z, Li Y, Wu J, et al: Loss of miR-204 expression enhances glioma migration and stem cell-like phenotype. Cancer Res 73: 990-999, 2013.

17. Malin S, McManus S and Busslinger M: STAT5 in B cell development and leukemia. Curr Opin Immunol 22: 168-176, 2010.

18. Blix ES, Irish JM, Husebekk A, et al: Phospho-specific flow cytometry identifies aberrant signaling in indolent B-cell lymphoma. BMC Cancer 12: 478, 2012.

19. Kirk R: Haematological cancer: Hit the lymphoma, JAK. Nat Rev Clin Oncol 9: 608, 2012 